\title{
La réforme ferroviaire française de 1997 : une histoire à rebondissements pour un scénario original
}

The French Railway Reform of 1997: the many twists of an original story

Arnaud Passalacqua

\section{(2) OpenEdition}

\section{Journals}

Édition électronique

URL : https://journals.openedition.org/rhcf/2122

DOI : $10.4000 /$ rhcf. 2122

\section{Éditeur}

Rails \& histoire

Édition imprimée

Date de publication : 2 avril 2014

Pagination : 83-103

ISSN : 0996-9403

\section{Référence électronique}

Arnaud Passalacqua, «La réforme ferroviaire française de 1997 : une histoire à rebondissements pour un scénario original », Revue d'histoire des chemins de fer [En ligne], 45 | 2014, mis en ligne le 29 mai 2015, consulté le 22 avril 2022. URL : http://journals.openedition.org/rhcf/2122 ; DOI : https://doi.org/ $10.4000 /$ rhcf. 2122 
Arnaud PASSALACQUA

\section{La réforme ferroviaire française de 1997 : une histoire à rebondissements pour un scénario original}

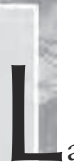

réforme ferroviaire française de 1997 est communément perçue comme une conséquence presque directe de la volonté européenne de transformer le système ferroviaire en un marché libéralisé. Dans les milieux ferroviaires, l'expression 91-4401 suffit à résumer ce qui est bien souvent ressenti comme une menace extérieure. Pourtant, la complexité de la réforme ellemême, de ses origines et des conditions dans lesquelles elle a été élaborée puis mise en place mérite un approfondissement*.

Derrière la directive 91-440, en effet, nombre d'éléments nationaux ont induit la nécessaire évolution du système ferroviaire français. Certains sont conjoncturels, comme le conflit de 1995, troisième grand mouvement social du xx ${ }^{e}$ siècle après 1936 et 1968, révélateur de bien des malaises. D'autres sont structurels, comme le poids de la dette ferroviaire, lié aux choix - ou plutôt aux non-choix - faits en termes de financement des lignes nouvelles à grande vitesse (LGV) et à la sous-estimation des coûts d'entretien du réseau.

\footnotetext{
* L'auteur tient à remercier Marie-Noëlle Polino et Jean-Louis Rohou pour la relecture de ce texte.

1- Il s'agit d'une allusion à la directive du Conseil du 29 juillet 1991 relative au développement de chemins de fer communautaires (91/440/CEE).
} 
Par ailleurs, cette réforme ne peut se résumer à la naissance de Réseau Ferré de France (RFF). Elle présente beaucoup d'autres aspects, notamment la régionalisation des transports, qui est probablement aujourd'hui l'une de ses grandes réussites.

Cette perception simplifiée de la réforme ferroviaire peut s'expliquer par la difficulté que l'on éprouve à bien comprendre la période troublée qui s'étend de l'automne 1995 au printemps 1998. Elle impose de porter attention à une chronologie fine marquée par plusieurs rebondissements, dont la consolidation de la réforme par son principal opposant. La richesse du contexte politique et syndical mérite également une attention particulière. C'est donc une analyse précise que cet article propose, des origines de la réforme à sa validation définitive par la "réforme de la réforme ", en cherchant d'abord à établir des faits afin d'éclairer notre compréhension de cet épisode fondamental de l'histoire ferroviaire ${ }^{2}$.

\section{5, un conflit révélateur}

Le 15 novembre 1995, Alain Juppé présenta le plan d'une réforme de la Sécurité sociale destinée à améliorer la situation financière de l'institution. Le Premier ministre proposait en particulier d'allonger la durée de cotisation des fonctionnaires et des salariés relevant des régimes spéciaux de 37,5 à 40 annuités pour obtenir une retraite à taux plein, ce qui était la norme pour les salariés du secteur privé depuis 1993. L’Assemblée nationale appuya le plan en votant la confiance à un gouvernement décidé à agir par ordonnances, tandis que très rapidement l'opposition dénonça ce qu'elle estimait être le démantèlement de la Sécurité sociale.

De leur côté, les syndicats se trouvèrent d'abord divisés, la CFDT se refusant à appeler à la grève en raison des éléments positifs qu'elle trouvait dans le plan Juppé, tandis que d'autres demandaient une riposte immédiate. Mais des tensions étaient également palpables entre opposants, la CGT et FO ne tombant pas d'accord sur l'organisation d'une journée commune de protestation.

2- Cet article se fonde notamment sur la campagne d'archives orales menée par l'AHICF, en particulier la collecte intitulée «Les dix premières années de Réseau ferré de France (RFF) », financée par RFF et successivement réalisée par Jean-Pierre Williot, Pierre Zembri et Arnaud Passalacqua. Les témoignages recueillis sont destinés à être publiés sur le site www.memoireorale.org. 
Le 24 novembre, une première journée d'action, consacrée à la mobilisation des fonctionnaires, rassembla de 500000 à un million de manifestants dans toute la France. Alors que la seconde n'était prévue que le 28 novembre à l'appel de FO, les syndicats cheminots - FGAAC, CGT, CFDT et FO décidèrent de poursuivre immédiatement le mouvement de grève. Ce fut alors l'entrée dans un long conflit social, qui ne s'acheva que le 15 décembre.

Au-delà du projet du Premier ministre, qui avait fait le choix de ne pas négocier avant de rendre ses décisions publiques (prévoyant donc probablement de reculer sur certains points) et de sa fermeté, l'ampleur du conflit dans le monde ferroviaire s'explique notamment par le fait que l'annonce coïncidait avec un élément spécifique aux relations entre l'État et la SNCF, la négociation du contrat de plan 1996-2000. Sur ce terrain, les relations entre le gouvernement et les syndicats s'étaient tendues, dans la mesure où la ligne directrice du projet de contrat consistait à maîtriser les coûts d'exploitation, à réduire le rythme des investissements et à céder des actifs. Jean-Claude Gayssot, ancien cheminot devenu député communiste, avait dénoncé le "véritable dépeçage de l'entreprise nationale ${ }^{3}$ " que rendaient possible, selon lui, le contrat de plan et la révision du cahier des charges de la SNCF qu'il envisageait.

C'est la conjonction de ces deux situations qui explique le poids particulier que prirent les cheminots dans le conflit de 1995 : allongement de la durée de cotisation avant la retraite et rattachement de leur caisse de retraite à la caisse générale, d'une part, perspective d'un contrat de plan de rigueur, d'autre part. Toutes deux trouvèrent un écho dans l'espace public : concernant la première, par le rôle que jouèrent les cheminots dans le mouvement de grève national (et la notion de grève par procuration), pour la seconde, ce fut la reprise, dans La Vie du rail, d'une carte présentant les tronçons du réseau ayant la plus faible utilité collective, vite traduite dans l'opinion publique comme des projets de fermeture de lignes ${ }^{4}$.

Face à l'ampleur d'un mouvement qui ne s'essoufflait pas et qui semblait trouver un écho plutôt favorable dans la population, malgré la capacité des grévistes de bloquer l'économie et les déplacements, le gouvernement se trouva contraint de faire machine arrière. En effet, contrairement à celle de 1986, la grève cheminote de 1995 fut soutenue par les voyageurs et se déroula

3- Assemblée nationale, séance du 25 octobre 1995.

4- Voir «Les grands choix du contrat de plan », La Vie du rail, n 2522 (29 novembre 1995), p. 4-13. Une première version de cette carte avait été publiée par la presse communiste, constituant un facteur initial de mobilisation. 
dans une ambiance où la solidarité semblait l'emporter sur l'agacement. Des pratiques nouvelles de mobilité en bénéficièrent même, rollers et trottinettes notamment, mais également le plus classique vélo.

À partir du 5 décembre, le gouvernement commença à modifier le plan Juppé dont plusieurs mesures étaient retirées. Le 10, Alain Juppé annonçait le report sine die de la signature du contrat de plan et, le 15, Jean Bergougnoux qui s'était fortement investi en sa faveur démissionna de la présidence de la SNCF. Le recul du gouvernement semble donc être total et la victoire des opposants éclatante.

Le paradoxe de la réforme ferroviaire de 1997 est qu'elle prend racine dans cette situation traumatisante pour les différents acteurs, même pour ceux qui en étaient sortis vainqueurs. Alors que le statu quo aurait dû s'imposer (les propositions d'évolution avaient été refusées), il est frappant de constater que le monde ferroviaire allait vivre en l'espace de deux ans sa plus grande réforme depuis 1937. Ce paradoxe se comprend mieux lorsqu'on analyse, au-delà du conflit de 1995, les différentes dimensions de ce monde ferroviaire qui, pour important qu'il soit, a surtout joué le rôle de révélateur d'un mal-être.

\section{L'enlisement du monde ferroviaire au milieu des années 1990}

À cette époque, le paysage ferroviaire français était effectivement marqué par l'existence de plusieurs zones sombres. La principale, aux yeux de nombre d'acteurs, peut-être parce que la plus simple à quantifier, était la situation financière dans laquelle se trouvait la SNCF. En 1996, la dette ferroviaire s'élevait à 237 milliards de francs (46 milliards d'euros ${ }^{5}$ ), dont 208 (41 milliards d'euros ${ }^{5}$ ) pour la seule SNCF. Pour un chiffre d'affaires de 55 milliards de francs ( 11 milliards d'euros ${ }^{5}$ ), le résultat d'exploitation accusait une perte de 466 millions de francs (91 millions d'euros $\left.{ }^{5}\right)$. Surtout, les pertes annuelles, dues à la charge de la dette et au déficit du compte d'infrastructure, s'élevaient à 15 milliards de francs (3 milliards d'euros ${ }^{5}$ ).

\section{Le poids de la dette et le déficit d'innovation}

Le contexte était morose, au-delà de la chute de fréquentation temporaire liée au conflit de 1995. Ainsi, en 1996, le trafic voyageurs avait baissé de 7,5 \% depuis 1990 et l'entrée du pays dans une crise économique. Les parts de marché de la SNCF, tant en voyageurs qu'en marchandises, étaient également en chute libre.

5- Les montants en euros sont exprimés en équivalent 2012. 
Au-delà de pertes d'exploitation somme toute assez faibles, la situation financière était donc bloquée par le poids de la dette. Une dette dont l'origine peut être attribuée aux dépenses réalisées par l'entreprise pour assurer le développement et l'entretien de son infrastructure. En effet, la SNCF s'était lourdement endettée pour la construction des LGV successivement ouvertes depuis 1981. Surtout, le compte d'infrastructure enregistrait des déficits réguliers du fait d'une sous-estimation chronique des coûts d'entretien et d'exploitation ${ }^{6}$.

L'exemple de la LGV Nord, inaugurée en 1993, est parlant. Alors que l'enquête publique prévoyait un taux de rentabilité interne économique de $12,9 \%$, ce taux ne fut estimé, en 2005 , qu’à $2,9 \%$ par le bilan $\mathrm{LOTI}^{7}$. La ligne ayant été entièrement financée par la SNCF, à l'exception de certains tronçons spécifiques, ces résultats bien plus faibles qu'attendu ont conduit la LGV Nord à peser lourdement sur les comptes de la SNCF. Objets largement politiques, les LGV n'avaient été prises en charge directement par les pouvoirs publics que dans une faible mesure, ce qui explique en partie l'explosion de la dette ferroviaire au milieu des années 1990. Mais l'État se trouvait finalement payeur par sa contribution annuelle à la SNCF, environ 50 milliards de francs (10 milliards d'euros ${ }^{5}$ ), également liée au poids des retraites cheminotes.

Cette énorme dette remettait ainsi en question le modèle ferroviaire français, à court terme puisque les banques se montraient de plus en plus réticentes à financer la SNCF mais également à long terme, dans la mesure où la France s'était engagée dans une ambitieuse politique de grande vitesse ferroviaire, inscrite dans le schéma directeur adopté par décret en $1992^{8}$. Le TGV était encore au cœur de la loi Pasqua de $1995^{\circ}$ et avait fait l'objet la même année de nombreuses promesses lors de la campagne présidentielle. La responsabilité de la construction des LGV dans l'endettement de la SNCF ne pouvait donc que conduire à une remise en question de ce paradigme. À l'automne 1995, la circulaire Idrac instaura des règles d'évaluation socio-éco-

6- «Le déficit du compte d'infrastructure est le facteur principal du déficit de la SNCF et, par voie de conséquence, de l'accroissement inexorable de son endettement ", in Hubert HaENEL, Avis fait au nom de la commission des Finances, du contrôle budgétaire et des comptes économiques de la Nation sur le projet de loi portant création de l'établissement public «Réseau ferré national », n 178, Sénat, 19961997, partie II, A.

7- Bilan LOTI de la LGV Nord, Paris, RFF, 2005, p. 5.

8- Décret $\mathrm{n}^{\circ}$ 92-355 du $1^{\text {er }}$ avril 1992 approuvant le schéma directeur national des liaisons ferroviaires à grande vitesse.

9- Loi nº 95-115 du 4 février 1995 d'orientation pour l'aménagement et le développement du territoire. 
nomique des projets, dans le sillage des travaux de Marcel Boiteux ${ }^{10}$, tandis que le gouvernement confiait à Philippe Rouvillois, ancien président de la SNCF, une mission d'étude d'un calendrier plus réaliste du schéma directeur.

D'autres pans de l'activité ferroviaire rencontraient également des difficultés. Si les accidents dramatiques du milieu des années 1980 étaient surmontés, les problèmes persistaient du côté d'un réseau classique vieillissant et de la relation avec les voyageurs, marquée notamment par le fiasco de la mise en place du système de réservation Socrate, en 1993. Des dépassements importants étaient également enregistrés dans le coût de l'un des projets les plus ambitieux, Éole. Plus généralement, c'est la gouvernance de la SNCF qui semblait malade. Jean Bergougnoux était un président à la légitimité contestée, tandis que ses propositions pour faire face aux difficultés financières étaient sans proportion avec le problème posé. Dans ce contexte, le contrat de plan 1996-2000 avait pu susciter un espoir qui fut déçu par les événements de l'automne 1995. Le conflit social a ainsi conduit la SNCF à exprimer les doutes qu'elle entretenait sur elle-même.

Contrairement à une RATP qui avait su renouveler sa structure, notamment sous l'impulsion de Christian Blanc, et s'ouvrir aux questions de prospective, la SNCF en demeurait éloignée, ce qui la conduisait à subir les évolutions. Ses terrains d'innovation touchaient essentiellement à la grande vitesse et à la sécurité des agents comme des voyageurs. Mais il n'y avait que peu d'anticipation des questions socio-économiques, malgré la création d'une direction de la stratégie en 1991.

Toutefois, depuis une dizaine d'années, un processus intéressant se développait au sein du monde ferroviaire : la construction d'une offre régionale de transport. À la SNCF, la volonté de plusieurs acteurs, comme Jacques Chauvineau ou Jacques Fournier, inspira un mouvement en faveur du renouveau des transports régionaux, notamment à partir de la création des TER, en 1987. On pensait également que les régions pouvaient disposer de financements dont l'État semblait toujours plus dépourvu. Dans une entreprise focalisée sur le TGV et sur la capacité de celui-ci à concurrencer l'avion, l'impulsion définitive en faveur des trains régionaux vint toutefois de l'extérieur, par le biais du rapport Haenel de $1994^{11}$.

10- Instruction cadre relative aux méthodes d'évaluation économique des grands projets d'infrastructure de transport jointe à la circulaire du 3 octobre 1995 du secrétaire d'État aux Transports. 11- Hubert HAENEL, Régions, SNCF : vers un renouveau du service public, Rapport au ministre de l’Équipement, des Transports et du Tourisme, 1994. 


\section{Le contexte européen}

Cette situation nationale du chemin de fer était rendue plus complexe par deux éléments à l'échelle européenne. Le premier est la directive 91-440 du 29 juillet $1991^{12}$. Ce texte imposait la séparation, dans les comptes des sociétés, entre gestion de l'infrastructure ferroviaire et fourniture de services de transport, sans exiger toutefois que ces deux activités soient assurées par deux entreprises distinctes. L'esprit de ce texte consistait à garantir, à terme, l'ouverture à la concurrence des réseaux de chemin de fer, comme dans les secteurs des télécommunications et de l'énergie. Il ne prévoyait l'ouverture qu'aux regroupements internationaux mais introduisait la notion de redevance pour l'utilisation des réseaux. Enfin, il autorisait le transfert de la dette ferroviaire aux États.

À la fin de 1995, cette directive avait été inégalement appliquée par les États membres, alors au nombre de 15. L'Allemagne avait repris une grande partie de la dette des chemins de fer en créant la Deutsche Bahn réunifiée en 1994. En France, la direction de la SNCF n'avait pas vraiment pris le texte en considération, craignant la concurrence et préférant développer des partenariats avec les opérateurs historiques, comme pour Thalys, créé en mai $1995^{13}$. La directive avait toutefois été débattue ${ }^{14}$, un décret de mai $1995^{15}$ en prévoyant la transposition par application de la séparation comptable, et était abordée dans les discussions autour du contrat de plan 1996-2000. En 1995, deux autres directives avaient par ailleurs renforcé l'incitation donnée par l'Union à organiser les services ferroviaires nationaux sous la forme de marchés ouverts à plusieurs opérateurs, en précisant les modalités de délivrance de licences ferroviaires et de répartition des sillons ${ }^{16}$. Sans qu'une causalité directe puisse être affirmée, le contexte européen était donc favorable à une réforme.

12- Directive du Conseil du 29 juillet 1991 relative au développement de chemins de fer communautaires (91/440/CEE).

13- Une partie de la dette de la SNCF avait néanmoins été sortie des comptes de l'entreprise par la création du Service Annexe d'Amortissement de la Dette (SAAD) en 1991. Cette structure, ambivalente, finit par être considérée comme gérant une dette publique en 2007.

14- Voir notamment Hervé de TréGLODÉ, « Le grand débat sur la séparation de l'infrastructure ", Revue générale des chemins de fer, vol. 110, n 3 (mars 1991), p. 5-9.

15- Décret no 95-666 du 9 mai 1995 portant transposition de la directive 91-440 du Conseil des Communautés européennes du 29 juillet 1991 sur le développement de chemins de fer communautaires et relatif à la gestion et l'utilisation de l'infrastructure du réseau ferré national. 16- Directive du Conseil du 19 juin 1995 concernant les licences des entreprises ferroviaires (95/18/CE) et directive du Conseil du 19 juin 1995 concernant la répartition des capacités d'infrastructure ferroviaire et la perception de redevances d'utilisation de l'infrastructure (95/19/CE). 
La deuxième contrainte européenne est liée à l'adoption du traité de Maastricht, signé en 1992, et à la notion de critères de convergence qu'il introduisait. En vue de l'établissement d'une monnaie unique, les États membres de la nouvelle Union européenne devaient effectivement faire converger leurs économies, en respectant cinq critères. Parmi ceux-ci figurait la limitation de la dette publique à $60 \%$ du produit intérieur brut. Or la France se trouvait aux abords de ce seuil, évalué en 1997. La dette de la SNCF n'était pas incluse dans la dette publique puisque l'entreprise, bien que publique, tirait plus de la moitié de ses recettes d'une activité marchande. La reprise de la dette ferroviaire par l'État menaçait donc le droit de la France à adopter l'euro. Ainsi, la dette demeurait un point négatif, malgré la sortie de la crise sociale de 1995.

\section{L'épisode Loïk Le Floch-Prigent, de la relance de la SNCF au discrédit de son patron}

Avec le départ de Jean Bergougnoux, la SNCF se trouvait sans président. Malgré quelques hésitations liées aux aléas judiciaires pesant sur lui, Loïk Le Floch-Prigent, patron de Gaz de France passé par un cabinet du gouvernement Mauroy, fut nommé le 20 décembre 1995. Dès lors, il lança l'entreprise dans une grande mutation, tout en s'efforçant de renouer le dialogue social. Il fit le choix d'élaborer un plan d'entreprise avant la renégociation du contrat de plan. Puis, il réorganisa la direction de la SNCF, essentiellement autour de deux pôles : les clientèles, confiées à Alain Poinssot, et l'exploitation, confiée à Francis Taillanter. Le directeur général Jean-François Bénard, démissionnaire, ne fut pas remplacé.

Loïk Le Floch-Prigent parvint à donner un nouveau visage à l'entreprise et à susciter une nouvelle ambiance chez les agents, en se voulant à la fois ferme, franc et dynamique. En se fondant sur son image de patron de gauche, il parvint à avancer des idées qui semblaient auparavant inaudibles. Il décida ainsi de se préoccuper de la rentabilité de l'activité, des recettes et non des coûts, et lança la SNCF à la reconquête de ses clients.

Mais, dès le printemps 1996, la presse bruissait de rumeurs sur son implication dans une affaire financière, lors de son passage à la tête d'Elf-Aquitaine. Dans la nuit du 4 au 5 juillet 1996, la juge Eva Joly le plaçait en détention provisoire, avant de perquisitionner dans les locaux de la SNCF. Ce n'est qu'après deux semaines de détention que Loïk Le Floch Prigent démissionna de son poste. Le 24 juillet 1996, la SNCF retrouva un patron en la personne de Louis Gallois, condisciple d'Alain Juppé à l'ENA, venu de l'Aérospatiale et déjà pressenti pour ce mandat à la fin de 1995. 


\section{L'élaboration de la réforme Pons-Idrac : la construction originale du nouveau paysage ferroviaire}

Parallèlement et plus discrètement, le gouvernement décidait d'aborder la réforme du système ferroviaire par un autre biais. Puisqu'Alain Juppé et Bernard Pons, alors ministre de l'Équipement, du Logement, des Transports et du Tourisme, craignaient de toucher à ce dossier que l'épisode de 1995 rendait brûlant, on le confia à une commission présidée par Claude Martinand, ingénieur des ponts et chaussées, directeur des Affaires économiques du ministère et ancien directeur du cabinet du communiste Charles Fiterman, dont était issue la LOTI. Formée de cinq membres, elle travailla extrêmement rapidement et rendit son rapport dès la fin février $1996^{17}$. Il posait plusieurs questions tout en jugeant que des décisions devaient être prises afin de sortir d'une forme d'enlisement.

\section{Du rapport au projet de loi}

Ce travail solide, qui abordait les problèmes de front, suscita un vaste débat national qui se déroula dans les conseils économiques et sociaux régionaux puis au Conseil économique et social. Différents acteurs apportèrent ainsi leurs réponses aux questions posées par la commission (direction de la SNCF, syndicats cheminots, élus, etc.). Cette forme originale contribua à renouveler le regard porté sur le monde ferroviaire, en sortant du cadre, discrédité par le conflit de 1995, des négociations préalables à un contrat de plan entre les seuls État et SNCF au niveau national. Sur le fond, le rapport proposait de distinguer le service public ferroviaire et la SNCF, qui, par ailleurs, ne devait pas être lésée par des responsabilités financières que l'État n'avait pas assumées. Au risque, toutefois, de dénier à la SNCF son rôle d'autorité organisatrice de fait.

Ce débat parvint à transformer le rapport de la commission en un véritable projet, appuyé sur plusieurs idées : clarification des relations entre l'État et la SNCF, nécessité d'un désendettement de la SNCF, engagement d'une régionalisation des transports et souci de la rentabilité des investissements futurs. Le débat s'acheva devant le Parlement en juin 1996. Le gouvernement y présenta les grandes lignes de la réforme qu'il souhaitait engager. D’abord, la création d'un nouvel établissement public chargé de "la responsabilité de l'infrastructure, [de] porter les dettes contractées [...] pour financer les infrastructures, [d'] assurer les investissements [et de] rémunérer la SNCF pour

17- Claude MARTINAND, Débat national sur l'avenir du transport ferroviaire : rapport introductif, Rapport au ministre de l'Équipement, du Logement, des Transports et du Tourisme, 1996. 
l'entretien et l'exploitation du réseau $»^{18}$. Sa rémunération était prévue par le moyen de péages dus par la SNCF. Bernard Pons rassura les cheminots quant à leur statut, qui était maintenu, et au caractère public de la SNCF, qui ne fut pas discuté. Ensuite, il s'agissait de développer la régionalisation des transports ferroviaires, par des transferts financiers de l'État vers les régions désireuses de prendre en main les services régionaux. Là aussi, le ministre précisa que ni l'unité du réseau ni celle de la SNCF n'étaient en cause. Fort du soutien des députés de l'écrasante majorité de droite, Bernard Pons affirmait ainsi préparer au mieux la SNCF aux défis qui l'attendaient, notamment ceux de la concurrence.

L'intérêt pour les sujets ferroviaires ne se démentait pas et les députés furent nombreux à désirer s'exprimer sur le sujet, chacun rappelant son amour du train. Si le clivage politique était net, d'autres logiques se dessinèrent. Ainsi, les Alsaciens-Lorrains se sont mobilisés dans ce débat, car ils se sentaient concernés à double titre, par le projet de LGV Est et par l'expérience de Métrolor. Il en fut de même des élus du Nord, peut-être plus sensibles au vent soufflant de Bruxelles. Dans les débats, la distinction entre la SNCF et le système ferroviaire n'était pas toujours bien nette. Une telle confusion régnait également en 1995. Sur le fond, les oppositions provenaient des bancs des socialistes, qui ne présidaient qu'une seule région à l'époque, et des communistes. La personnalité du communiste Jean-Claude Gayssot domina alors les débats, du fait de son autorité d'ancien cheminot. Les craintes principales touchaient à des arrière-pensées qu'aurait entretenues le gouvernement : privatisation totale ou partielle de la SNCF, mise en cause du statut des agents, ouverture à la concurrence, etc.

Le projet de loi correspondant fut élaboré au sein du cabinet d'AnneMarie Idrac, secrétaire d'État chargée des Transports. Olivier Grunberg et Frédéric Baverez y travaillèrent en lien avec Hubert du Mesnil, directeur des Transports terrestres, et David Azéma, conseiller de Louis Gallois. Pendant l'été, l'influence européenne se fit sentir, avec la présentation par le commissaire aux transports Neil Kinnock d'un Livre blanc ${ }^{19}$ qui ouvrait une phase de libéralisation du transport ferroviaire - totale pour le fret, partielle pour les voyageurs. Afin de rassurer les syndicats français, Bernard Pons et Anne-Marie Idrac affirmèrent leur opposition à ce texte, au risque d'isoler la France.

18- Assemblée nationale, séance du 14 juin 1996.

19- Une stratégie pour revitaliser les chemins de fer communautaires, Communauté européenne, Bruxelles, 1996. 


\section{Du projet à la loi}

Septembre vit l'adoption d'un pacte de modernisation, qui tint lieu de contrat de plan entre la SNCF et l'État. Dans ce cadre, l'entreprise s'engageait sur plusieurs points : équilibre global dès 1998 et de chacune de ses activités en 2000, réduction des charges d'infrastructure, hausse progressive des péages à partir de 1999, etc. Louis Gallois présenta les trois axes qu'il retenait pour dynamiser la SNCF : " le client, la régionalisation et l'allégement des structures de direction ${ }^{20}$. "Il annonça également le déménagement du siège de l'entreprise qui quittait le site historique du PLM, 88, rue Saint-Lazare. Au cours de l'automne, il avançait donc dans la définition d'un plan industriel préfigurant la gestion par activités. La période fut également marquée pour la SNCF par la remise du rapport Rouvillois ${ }^{21}$, qui préconisait de revoir à la baisse l'ambition des projets de LGV. Les discussions se poursuivirent également sur les choix entre train pendulaire sur voie classique et TGV, tandis que la réalisation de la LGV Est cherchait toujours un calendrier.

Parallèlement, le climat social s'alourdit dans différents secteurs, dont le monde ferroviaire. Des grèves régionales touchèrent la $\mathrm{SNCF}$, témoignant de diverses inquiétudes. Surtout, la CGT et la CFDT s'opposaient au projet de réforme proposé par le gouvernement, tandis que les petits syndicats (FO, CFTC et FMC) y étaient plutôt favorables. Le conseil des ministres adopta le projet de loi le 16 octobre, veille d'une journée de mobilisation nationale des fonctionnaires et agents des services publics.

La grogne montait, non sans rappeler 1995. Le 28 octobre, la CGT demanda le retrait du projet de loi. Le gouvernement préférait ne pas précipiter les choses et tenta de déminer le terrain ferroviaire en annonçant, le 4 novembre, le report de la discussion du projet de loi. Le rapporteur de la loi au Sénat, le RPR François Gerbaud, qui avait formulé des demandes d'approfondissement, fut mandaté pour poursuivre la concertation. La possibilité d'une confusion avec le Livre blanc européen, malgré la position de la France qui lui était hostile, encourageait également ce report.

20- Louis GaLLoIs, «Les "trois moteurs” pour remobiliser l'entreprise », La Tribune, 3 septembre 1996.

21- Philippe Rouvillois, Rapport sur les perspectives en matière de création de nouvelles lignes ferroviaires à grande vitesse, Rapport au ministre de l'Équipement, du Logement, des Transports et du Tourisme, 1996. 
Dans un communiqué, le parti socialiste dénonça alors ce qui n'était à ses yeux " qu'un montage sommaire, précipitamment élaboré pour mettre un terme au grand mouvement de protestation de décembre 1995 en contrepartie d'une promesse de reprise d'une partie de la dette de la SNCF ${ }^{22}$. Pour sa part, Bernard Pons tint à assurer que ce qui s'appelait alors RFN (Réseau Ferré National) verrait bien le jour. Au même moment, Alain Juppé confiait à Claude Martinand une mission de préfiguration.

Tandis que les mouvements de grève des cheminots ne retrouvaient pas l'ampleur de ceux de l'année précédente, c'est plutôt " au son du camion ", selon le nom de l'opération organisée pour bloquer le périphérique parisien le 7 novembre 1996, que se tendait le climat social. Les routiers, qui revendiquaient une détaxe sur le carburant diesel, exercèrent en se mobilisant une puissante pression sur le gouvernement. Mais, sur le front ferroviaire, les esprits se calmèrent jusqu’au début de 1997.

\section{Le vote de la loi}

L'autre volet de la réforme, la régionalisation, était peu discuté dans les médias et avançait parallèlement. L'idée d'une expérimentation par des régions volontaires faisait son chemin. Rhône-Alpes, Provence-Alpes-Côte d'Azur, Centre, Pays de la Loire, Nord - Pas-de-Calais et Alsace voulaient tenter l'aventure, avec la possibilité de revenir en arrière. Le 22 novembre, Rhône-Alpes approuva ainsi les conventions instituant la régionalisation à partir du $1^{\text {er }}$ janvier 1997.

Avant le passage de la loi au Sénat, le gouvernement engagea des discussions avec les différents partenaires, notamment les organisations sociales. De façon exceptionnelle, elles ont abordé les projets de décrets d'application concernant les missions de la nouvelle structure chargée du réseau, la constitution de son patrimoine et les redevances payées par la SNCF. La CGT, déstabilisée par l'épisode Le Floch-Prigent, n'avait plus alors l'assurance qui était la sienne au sortir du conflit de 1995. Bernard Pons rejeta son exigence d'organiser un référendum auprès des cheminots. La CFDT était alors l'opposant le plus hostile au projet, aux côtés du jeune syndicat Sud-Rail. Ainsi, des grèves ont ponctué la discussion du projet au Parlement mais elles ne rencontrèrent qu'un assez faible succès. Simultanément et probablement en accord avec le gouvernement, Louis Gallois présenta un bilan très négatif pour 1996 - 15 milliards de francs (3 milliards d'euros ${ }^{5}$ ) de pertes -, ce qui renforça la conviction de la nécessité d'une réforme.

22- «SNCF - Les députés PS dénoncent le projet de réforme », Reuters, 6 novembre 1996. 
Après avoir convaincu Jacques Chirac, Bernard Pons, qui connaissait bien l'Assemblée nationale, choisit de présenter le texte d'abord au Sénat, où il était moins sûr de ses appuis. Son expérience s'est avérée décisive dans l'adoption d'un texte ambitieux dans un contexte aussi délicat. Il reçut notamment les principaux parlementaires des différents groupes au préalable. Dans les deux chambres, la discussion donna la possibilité à la gauche d'exprimer vivement son désaccord. Les débats se retrouvaient d'ailleurs très largement dans la presse. La principale figure de l'opposition à la réforme restait Jean-Claude Gayssot, qui annonça notamment que « les cheminots et les usagers sauront apporter les réponses appropriées pour mettre en échec cette loi ${ }^{23}$. Au parti socialiste, Ségolène Royal, en charge des services publics, tint un discours similaire, affirmant que la réforme ne résolvait rien et promettant de "[réinstaurer] d'abord l'unité de la SNCF $»^{24}$.

Le texte était néanmoins voté au Sénat le 24 janvier 1997, après l'adoption de quelques amendements, notamment celui proposé par la commission des affaires économiques qui réévaluait le transfert de dette de 125 milliards de francs $\left(24\right.$ milliards d'euros $\left.{ }^{5}\right)$ à 134 milliards $\left(26\right.$ milliards d'euros $\left.{ }^{5}\right)$. À l'Assemblée, le texte fut voté le 7 février, dans une version conforme puisque la majorité ne proposa pas d'amendements tandis que près de 400 amendements et sous-amendements l'étaient par la gauche. C'était un succès de procédure pour le gouvernement.

\section{Une loi et trois décrets : la réforme ferroviaire}

La loi fut finalement promulguée le 13 février 1997, avec effet rétroactif au $1^{\text {er janvier }}{ }^{25}$. Elle créait l'établissement public RFF, l'État restant en charge de la définition du réseau. Les missions de la SNCF, telles que définies par la LOTI, étaient revues en conséquence, puisqu'elle n'avait désormais plus la charge " de développer [...] le réseau ferré national ".

La loi précisait immédiatement que la SNCF assurait « la gestion du trafic et des circulations sur le réseau ferré national ainsi que le fonctionnement et l'entretien des installations techniques et de sécurité " pour le compte de RFF. Une convention devait régler les relations entre la SNCF et RFF dans ce

23- «Le Parlement adopte la réforme de la SNCF », La Tribune, 10 février 1997.

24- Voir «SNCF : les socialistes promettent la contre-réforme. Mise au point de Ségolène Royal entre grève et débat à l'Assemblée », Libération, 5 février 1997.

25- Loi n 97-135 du 13 février 1997 portant création de l'établissement public « Réseau ferré de France » en vue du renouveau du transport ferroviaire. 
domaine. Pour sa part, RFF était institué maittre d'ouvrage des projets mais pouvait confier des mandats à la SNCF.

Ce n'est que dans son article 5 que le texte abordait les transferts de patrimoine en faveur de RFF. La SNCF, qui n'était qu'affectataire d'un patrimoine appartenant à l'État, en était dessaisie au profit de RFF qui en obtenait la "pleine propriété ». Le patrimoine fut défini conformément à la directive 91-440, RFF obtenant:

les biens constitutifs de l'infrastructure [qui] comprennent les voies, y compris les appareillages fixes associés, les ouvrages d'art et les passages à niveau, les quais à voyageurs et à marchandises, les triages et les chantiers de transport combiné, les installations de signalisation, de sécurité, de traction électrique et de télécommunications liées aux infrastructures, les bâtiments affectés au fonctionnement et à l'entretien des infrastructures.

Pour sa part, la SNCF maintenait notamment son parc de logement social; elle conservait en effet :

les biens dévolus à l'exploitation des services de transport, qui comprennent les gares, les entrepôts et cours de marchandises ainsi que les installations d'entretien du matériel roulant, et [...] les ateliers de fabrication, de maintenance et de stockage des équipements liés à l'infrastructure, ainsi que les immeubles administratifs [et les] biens affectés au logement social ou au logement [de ses agents] par nécessité de service et [...] ceux affectés aux activités sociales, des filiales et des participations financières.

Larticle 7 précisait ensuite la contrepartie de cette dotation en patrimoine : RFF inscrivait à son passif une dette de 134 milliards de francs (26 milliards d'euros ${ }^{5}$ ). L'article 13 déterminait les ressources de RFF, notamment les redevances pour l'utilisation de son réseau, tout en indiquant que RFF n'en fixait pas lui-même le montant : celui-ci répondait à des contraintes de coût d'usage de l'infrastructure mais devait aussi permettre d'orienter la politique des transports.

Enfin, l'article 15 introduisit la régionalisation en faisant des régions volontaires des " autorités organisatrices des services régionaux de voyageurs " ferroviaires, contre compensation de charges transférées par l'État. Ce dispositif était expérimental et devait prendre fin au 31 décembre 1999.

Ce texte appelait donc des précisions, elles lui furent apportées par des décrets d'application pris dans un contexte politique exceptionnel : le 21 avril 1997, Jacques Chirac annonçait la dissolution de l'Assemblée nationale. Dès lors, ils furent très rapidement signés, le 5 mai.

Le premier décret ${ }^{26}$ est le plus original dans la mesure où son article 4 prévoit que « RFF ne peut accepter un projet d'investissement sur le réseau

26- Décret n 97-444 du 5 mai 1997 relatif aux missions et aux statuts de Réseau ferré de France. 
ferré national, inscrit à un programme à la demande de l'État, d'une collectivité locale ou d'un organisme public local ou national, que s'il fait l'objet de la part des demandeurs d'un concours financier propre à éviter toute conséquence négative sur les comptes de RFF sur la période d'amortissement de cet investissement ". Il s'agit bien là de la prise en compte des déboires financiers liés aux LGV. L'idée était d'éviter que la réalisation de projets d'infrastructure vienne de nouveau accroître la dette ferroviaire, en plaçant les pouvoirs publics face à leurs responsabilités. Cette disposition, vite connue sous le nom d'article 4, est l'une des principales innovations de la réforme de 1997.

Le deuxième texte ${ }^{27}$ 's attelait à un problème qui devait s'avérer épineux, la répartition du patrimoine entre la SNCF et RFF. Une commission nationale de répartition des actifs fut prévue ${ }^{28}$. Pour les cas problématiques, elle devait formuler des propositions, sur lesquelles le gouvernement se prononcerait. Le décret vint apporter des précisions à la loi, en détaillant les biens constitutifs du patrimoine ${ }^{29}$.

Enfin, le dernier décret ${ }^{30}$ détaillait le mécanisme des redevances, en indiquant qu'elles seraient composées « d'un terme forfaitaire correspondant à l'accès à [une] section pour une période donnée, d'un terme correspondant à la réservation d'une capacité d'infrastructure, exigibles même si cette capacité n'est pas utilisée, et d'un terme correspondant à la circulation effective ". Il fixait également le montant maximum des péages exigibles par RFF pour 1997 et 1998, dans le but de rassurer la SNCF.

Très rapidement, l'établissement public fut constitué. Le 14 mai, Claude Martinand était nommé à la tête de RFF, conférant à la nouvelle entité une dimension clairement ferroviaire, alors que Bercy aurait souhaité y placer l'un de ses fonctionnaires.

\section{Un nouveau rebondissement : Jean-Claude Gayssot et la " réforme de la réforme "}

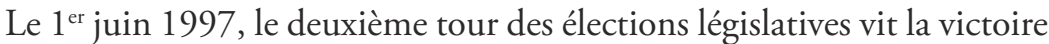
de la gauche plurielle, sous la conduite de Lionel Jospin. Jean-Claude Gayssot avait été l'un des négociateurs de l'accord conclu pour ces élections entre les

27- Décret $n^{\circ}$ 97-445 du 5 mai 1997 portant constitution du patrimoine initial de l'établissement public Réseau ferré de France.

28- Elle ne fut réellement créée qu'en octobre 1998, Jacques Négrier, conseiller d'État honoraire, étant nommé à sa tête.

29- Ce partage ne s'est achevé qu'en 2006, après mise en demeure du gouvernement.

30- Décret n 97-446 du 5 mai 1997 relatif aux redevances d'utilisation du réseau ferré national. 
partis communiste et socialiste. Il se trouvait donc être l'un des communistes les plus en vue de cette majorité et obtint de larges attributions en devenant ministre de l'Équipement, des Transports et du Logement, ce qui traduisait également une volonté d'apaisement après le conflit de 1995. Dès lors, l'un des dossiers auquel il fut confronté en priorité a été la réforme ferroviaire. Jouant sur son image, il rassura en faisant valoir qu'il ne saurait être l'homme de la privatisation de la SNCF. Mais il ne s'engagea pas à effectuer un retour en arrière, préférant amorcer une phase préalable de consultation des partenaires. La CGT, la CFDT et Sud-Rail firent cependant pression sur le ministre en demandant l'abrogation de la réforme.

Pourtant, dès le 30 juin, les premiers articles laissant entendre que la réforme ne serait pas abrogée parurent dans la presse ${ }^{31}$. Il ne s'agirait que d'une " réforme de la réforme ". Les faibles démentis apportés par le ministère firent comprendre que ces fuites visaient à tester la sensibilité des partenaires sociaux. Début juillet, une solution s'esquissait, elle consistait à augmenter le désendettement de la SNCF - qui avait toujours semblé trop faible à JeanClaude Gayssot - et à créer une structure chapeautant RFF et la SNCF, afin de rendre au paysage ferroviaire l'unité réclamée par les syndicats. Le 10 juillet, le ministre annonça que la SNCF bénéficierait d'un nouveau désendettement de 20 milliards de francs $\left(4 \text { milliards d'euros }{ }^{5}\right)^{32}$, en contrepartie duquel elle devrait embaucher 1000 agents supplémentaires et 1000 employés sous contrats jeunes ${ }^{33}$, l'un des dispositifs phares du gouvernement. Implicitement, ces mesures correspondent à un maintien de la réforme.

Ce n'est qu'à l'automne que des tensions se firent de nouveau jour. Mais la revendication d'un retour en arrière abolissant la réforme, qui divisait les syndicats, se perdit parmi d'autres motifs de mobilisation plus unitaires, comme les salaires ou le temps de travail, dans le contexte du passage aux 35 heures. En témoigne la grève du 8 octobre, au succès mitigé. Le jeu syndical était également rendu complexe par l'approche des élections professionnelles de mars 1998 : la CGT voulait montrer sa fermeté et sa présence sur le terrain pour éviter de se faire déborder par Sud-Rail.

Le 20 octobre 1997, Jean-Claude Gayssot relança le débat en ouvrant la concertation sur la "réforme de la réforme ". Il affirme son " attachement à une certaine séparation entre les fonctions de propriétaire des infrastructures et les fonctions de gestionnaire et de transporteur, la situation antérieure

31- Voir «La réforme de la SNCF sera maintenue », La Tribune, 30 juin 1997.

32- Ce désendettement est porté fin 1997 à 28 milliards qui sont confiés au SAAD.

33- Il s'agit d'un contrat de travail réservé aux jeunes de 16 à 25 ans, une partie des cotisations sociales de l'employeur étant prise en charge par l'État. 
n'ayant pas fait la preuve de son efficacité ${ }^{34}$. Pour sa part, la CGT proposait la création d'une direction des infrastructures au sein du ministère, intégrant les différents modes de transport et rendant redondante l'existence de RFF. Jean-Claude Gayssot tenta par ailleurs de modifier les termes du débat en envisageant plus largement la question des relations entre les modes de transport. Il évoqua même l'idée de modifier la LOTI ${ }^{35}$.

Ce n'est que le 19 juin 1998, après de longs mois et une dernière grève, le 13 mai, où l'unité des syndicats s'était faite sur les revendications salariales et la réduction du temps de travail et non sur la réforme ferroviaire, que le ministre annonça sa décision. Il maintenait RFF, augmentait la participation financière de l'État, à hauteur de 37 milliards de francs (7 milliards d'euros $\left.{ }^{5}\right)$ sur trois ans et créait un Conseil supérieur du service public ferroviaire, organisme consultatif. Évidemment les syndicats hostiles à la réforme furent déçus par cette issue, n'attendant rien de ce " comité Théodule " ${ }^{36}$, mais leur position n'était pas assez forte pour mobiliser les cheminots contre cette " réforme de la réforme ».

Pourquoi Jean-Claude Gayssot n'est-il pas revenu sur la réforme engagée par ses prédécesseurs ? Il semble d'abord qu'il n'en ait pas trouvé le moyen. Contraint par les critères de Maastricht, qu'il entendait respecter, et malgré les recettes constituées par l'ouverture du capital de France Telecom (transformée en société anonyme aux actionnaires privés en 1996), le gouvernement ne pouvait pas reprendre la dette ferroviaire à son compte. Personne ne voulait non plus d'un retour de la dette dans les comptes de la SNCF. Par ailleurs, parmi les groupes d'intérêt favorables au chemin de fer, plusieurs s'exprimaient pour le maintien de la réforme, comme la FNAUT ${ }^{37}$. Pour sa part, la régionalisation ne posait pas de véritable problème, d'autant moins après les victoires de la gauche dans plusieurs régions en 1998.

Surtout, la longue durée du processus de concertation - qu'elle ait été voulue ou non - a conduit à ce que la réforme avance d'elle-même. C'est ainsi que RFF s'est installé, même si son fonctionnement fut brièvement bloqué à l'arrivée du nouveau gouvernement. Dès le 11 juillet 1997, son conseil d'administration approuva son premier budget, incluant une dotation de l'État de

34- «SNCF - Jean-Claude Gayssot laisse les syndicats sur leur faim », Les Échos, 21 octobre 1997.

35- "Jean-Claude Gayssot veut remanier la loi sur les transports intérieurs », Les Échos, $1^{\text {er }}$ octobre 1997.

36- « Réforme SNCF/Les syndicats refusent le "comité Théodule" », Reuters, 19 juin 1998.

37- Voir «Les usagers favorables à la poursuite des réformes ferroviaires », Les Échos, 7 juillet 1997. 
8 milliards de francs (2 milliards d'euros ${ }^{5}$ ) pour 1997. À la rentrée, la convention de gestion liant la SNCF et RFF était approuvée par les deux entités. Très vite, RFF se construisit également une image dans le monde financier, obtenant par exemple en septembre la notation AAA de l'agence Standard \& Poor's. Enfin, son président commença à exister dans les médias et des tensions apparurent entre Louis Gallois et lui, notamment à propos du montant des redevances d'infrastructure. De son côté, la SNCF entérinait également la réforme. Le $1^{\text {er }}$ janvier 1998, elle clarifia son organisation en distinguant la direction de l'infrastructure, en charge de l'exploitation et de la maintenance du réseau, et la direction de l'ingénierie, en charge des activités d'étude et de maitrise d'ouvrage. Le monde ferroviaire avait donc bien basculé.

\section{Conclusion}

Ainsi, la réforme ferroviaire de 1997 peut être considérée comme réalisée malgré un gouvernement qui ne voulait plus toucher au sujet très sensible des chemins de fer et un opposant devenu ministre. Ces conditions complexes ont donné naissance à une solution subtile qui évitait un découpage trop marqué de la SNCF, tout en créant la possibilité d'une nouvelle gouvernance du transport ferroviaire et de ses projets. Le poids du contexte national explique sans doute que la France ait construit cette solution originale au regard de celles qu'ont mises en œuvre les autres pays européens.

Le contexte européen ne peut en fait éclairer que très partiellement la réforme en question ici. Elle mérite probablement une analyse historique détaillée - que cet article ne fait qu'ébaucher - afin de mieux saisir les positions précises des acteurs. Peut-être devrait-elle être décrite comme la réforme ferroviaire de 1995-1998, voire de 1991-1998 ? Encore ne s'agit-il là que de la naissance de la réforme : on peut en effet considérer que sa mise en place a pris elle aussi une dizaine d'années, à l'image du dossier de la répartition du patrimoine entre la SNCF et RFF. La jeune entité a également connu ellemême des réformes de structure - en 2000 pour la principale - et a dû évoluer pour assumer de nouvelles missions, notamment la responsabilité d'attribuer les sillons sur le réseau, à partir de 2003. De son côté, la SNCF n'est pas demeurée immobile depuis la fin des années 1990 et a su s'adapter à un contexte nouveau pour moderniser l'offre de transport, tandis que l'Europe poursuivait son incitation à l'ouverture des marchés ferroviaires, par le biais des "paquets ferroviaires " successifs. Enfin, au-delà des débats qui peuvent exister entre la SNCF et RFF - qui sont bien compréhensibles au vu de leur histoire - il est important de rappeler que la réforme ferroviaire de 1997 a ouvert la voie à un 
partage des questions ferroviaires qui sont sorties du giron de l'État par l'ouverture aux régions, que ce soit par le biais de la régionalisation ou par celui de leur participation au financement des LGV. À cet égard, la LGV Est fut la première LGV issue d'une réforme qui était liée, en partie, aux dettes laissées par ses aînées.

\section{Documents cités}

\section{Articles de revue}

Tr égl odé Hervé (de), "Le grand débat sur la séparation de l'infrastructure ", Revue générale des chemins de fer, vol. 110, $\mathrm{n}^{\circ} 3$ (mars 1991), p. 5-9.

\section{Articles de presse, dépêches}

"Les grands choix du contrat de plan ", La Vie du rail, n 2522 (29 novembre 1995), p. 4-13.

"Les "trois moteurs" pour remobiliser l'entreprise ", La Tribune, 3 septembre 1996.

"SNCF - Les députés PS dénoncent le projet de réforme ", Reuters, 6 novembre 1996.

"SNCF : les socialistes promettent la contre-réforme. Mise au point de Ségolène Royal entre grève et débat à l'Assemblée ", Libération, 5 février 1997.

"Le Parlement adopte la réforme de la SNCF ", La Tribune, 10 février 1997.

"La réforme de la SNCF sera maintenue ", La Tribune, 30 juin 1997.

"Les usagers favorables à la poursuite des réformes ferroviaires ", Les Échos, 7 juillet 1997.

" Jean-Claude Gayssot veut remanier la loi sur les transports intérieurs ", Les Échos, $1^{\mathrm{er}}$ octobre 1997.

"SNCF - Jean-Claude Gayssot laisse les syndicats sur leur faim ", Les Échos, 21 octobre 1997.

"Réforme SNCF/Les syndicats refusent le "comité Théodule" ", Reuters, 19 juin 1998.

\section{Documents ministériels ou parlementaires}

Assemblée nationale, compte rendu de la séance du 25 octobre 1995, consultable (mai 2013) à l'adresse : http://archives.assemblee-nationale. fr/index.asp

Assemblée nationale, compte rendu de la séance du 14 juin 1996, en cours de numérisation. 
Bilan LOTI de la LGV Nord, Paris, RFF, 2005, consultable en ligne (mai 2013). Haenel Hubert, Avis fait au nom de la commission des Finances, du contrôle budgétaire et des comptes économiques de la Nation sur le projet de loi portant création de l'établissement public "Réseau Ferré National ", n 178, Sénat, 1996-1997, consultable (mai 2013) en ligne.

- Régions, SNCF : vers un renouveau du service public, Rapport au ministre de l'Équipement, des Transports et du Tourisme, 1994, consultable (mai 2013) en ligne.

Mar tinand Claude, Débat national sur l'avenir du transport ferroviaire : rapport introductif, Rapport au ministre de l'Équipement, du Logement, des Transports et du Tourisme, La Documentation Française, 1996, consultable (mai 2013) en ligne.

Livre Blanc Une stratégie pour revitaliser les chemins de fer communautaires, Communauté européenne, Bruxelles, 1996, consultable (mai 2013) en ligne.

Rouvill ois Philippe, Rapport sur les perspectives en matière de création de nouvelles lignes ferroviaires à grande vitesse, Rapport au ministre de l'Équipement, du Logement, des Transports et du Tourisme, La Documentation Française, 1996, consultable (mai 2013) en ligne.

\section{Documents à caractère juridique}

\section{France}

Loi n 95-115 du 4 février 1995 d'orientation pour l'aménagement et le développement du territoire.

Loi n 97-135 du 13 février 1997 portant création de l'établissement public "Réseau ferré de France " en vue du renouveau du transport ferroviaire.

Décret $\mathrm{n}^{\circ}$ 92-355 du $1^{\text {er }}$ avril 1992 approuvant le schéma directeur national des liaisons ferroviaires à grande vitesse

Décret $\mathrm{n}^{\circ}$ 95-666 du 9 mai 1995 portant transposition de la directive 91440 du Conseil des Communautés européennes du 29 juillet 1991 sur le développement de chemins de fer communautaires et relatif à la gestion et l'utilisation de l'infrastructure du réseau ferré national.

Décret n ${ }^{\circ}$ 97-444 du 5 mai 1997 relatif aux missions et aux statuts de Réseau ferré de France.

Décret $n^{\circ}$ 97-445 du 5 mai 1997 portant constitution du patrimoine initial de l'établissement public Réseau ferré de France.

Décret no 97-446 du 5 mai 1997 relatif aux redevances d'utilisation du réseau ferré national. 
Instruction cadre relative aux méthodes d'évaluation économique des grands projets d'infrastructure de transport jointe à la circulaire du 3 octobre 1995 du secrétaire d'État aux Transports.

\section{Europe}

Directive du Conseil du 29 juillet 1991 relative au développement de chemins de fer communautaires (91/440/CEE).

Directive du Conseil du 19 juin 1995 concernant les licences des entreprises ferroviaires (95/18/CE).

Directive du Conseil du 19 juin 1995 concernant la répartition des capacités d'infrastructure ferroviaire et la perception de redevances d'utilisation de l'infrastructure (95/19/CE). 
